\title{
Subunit Heterogeneity of Cytoplasmic Dynein: Differential Expression of 14 kDa Dynein Light Chains in Rat Hippocampus
}

\author{
Jen-Zen Chuang, ${ }^{1}$ Teresa A. Milner, ${ }^{2}$ and Ching-Hwa Sung ${ }^{1,3}$ \\ ${ }^{1}$ Department of Ophthalmology, The Margaret M. Dyson Research Institute, ${ }^{2}$ Department of Neurology and \\ Neuroscience, and ${ }^{3}$ Department of Cell Biology and Anatomy, Weill Medical College of Cornell University, New York, New \\ York 10021
}

Cytoplasmic dynein is a multi-subunit protein complex in which each subunit is encoded by a few genes. How these subunit isoforms are assembled and regulated to mediate the diverse functions of cytoplasmic dynein is unknown. We previously have shown that two highly conserved $14 \mathrm{kDa}$ dynein light chains, Tctex-1 and RP3, have different cargo-binding abilities. In this report, coimmunoprecipitation revealed that Tctex-1 and RP3 were present in mutually exclusive dynein complexes of brain. Two specific antibodies were used to examine the localization of these two dynein light chains in adult rat hippocampal formation and cerebral cortex. By light microscopy, Tctex-1 and RP3 immunoreactivities exhibited distinct and almost complementary distribution patterns in both brain regions. In hippocampal formation, Tctex-1 immunoreactivity was most enriched in somata of newly generated granule cells and scant in

Neurons rely extensively on microtubules (MTs) and MT motors for relaying cellular components to and from the soma and distal processes. The MTs in axons are organized with their minus-ends facing perikarya and their plus-ends facing synapses (Baas et al., 1988). The two types of MT motors, kinesin and cytoplasmic dynein, thus are believed to move molecules and membrane organelles in an anterograde and retrograde direction, respectively (Vallee and Bloom, 1991). Nevertheless, how these molecular motors mediate and regulate the transport of a variety of proteins and membrane organelles is poorly understood.

Unlike the large kinesin superfamily, only a few genes that encode dynein subunits have been described previously (Hirokawa et al., 1998), suggesting that other mechanisms must exist to regulate dynein activity and cargo specificity. One potential mechanism regulating dynein specificity is subunit heterogeneity of the dynein complex. Each dynein complex consists of two heavy chains (Vallee and Shpetner, 1990), two or three intermediate chains, a group of light intermediate chains (Gill et al., 1994), and several light chains (King et al., 1996a,b; Bowman et al., 1999). The dynein heavy chain contains ATPase and motor activity (Vallee and Shpetner, 1990). Several of the remaining

Received March 8, 2001; revised April 19, 2001; accepted May 8, 2001.

This work was supported by Career Development Award, The Dolley Green Special Scholar Award (Research to Prevent Blindness), The Foundation Fighting Blindness, National Institutes of Health (NIH) Grant EY11307 (C.-H. S.), and NIH Grants DA08259 and HL18974 (T.A.M.). We thank Kai Xu for technical help with Figure $1 B$.

Correspondence should be addressed to Dr. Ching-Hwa Sung, The Margaret M. Dyson Vision Research Institute, Weill Medical College of Cornell University, 1300 York Avenue, New York, NY 10021. E-mail: chsung@mail.med.cornell.edu. Copyright (C) 2001 Society for Neuroscience $0270-6474 / 01 / 215501-12 \$ 15.00 / 0$ the mature granule and pyramidal cell somata. In contrast, RP3 immunoreactivity was abundant in pyramidal and granule cell somata. Ultrastructural analysis of the dentate gyrus revealed both dynein light chains were associated with various membranous organelles that often were affiliated with microtubules. In addition, Tctex-1 and RP3 immunoreactivities were preferentially and highly enriched on membranous organelles and/or vesicles of axon terminals and dendritic spines, respectively. These results suggest that dynein complexes with different subunit composition, and possibly function, are expressed differentially in a spatially and temporally regulated manner. Furthermore, Tctex-1 and RP3 may play important roles in synaptic functions.

Key words: cytoplasmic dynein light chain; Tctex-1; RP3; hippocampus; newly born neuron; synaptic terminal dynein subunits are likely to function as adapters between cargoes and motors by direct and/or indirect interactions with cargo molecules (Vaughan and Vallee, 1995; Echeverri et al., 1996; Tai et al., 1999). The ability of different dynein subunits to interact specifically with different cargo molecules may allow for the binding of the dynein complex to its cargo to be regulated selectively. Moreover, multiple isoforms of nearly all of the dynein subunits have been identified (Gibbons et al., 1994; Tanaka et al., 1995; Criswell et al., 1996; Vaisberg et al., 1996). Although it is not clear how different isoforms of dynein subunits are assembled into the dynein complex, dyneins containing different subunit isoforms may mediate distinct subsets of the functions of dynein.

There are two known $14 \mathrm{kDa}$ dynein light chains (DLCs), Tctex-1 and RP3. Stoichiometric studies suggest that each dynein complex contains two 14 kDa DLCs (King et al., 1998). However, whether each dynein complex contains one of each isoform or two of the same isoform is unknown. Despite their similarity $(52 \%$ identity and $75 \%$ similarity at the amino acid level), Tctex- 1 and RP3 appear to display specific and differential binding to dynein cargo, suggesting that these two DLCs may be involved in different subsets of dynein function (Tai et al., 1999). To understand better the assembly of DLCs into the dynein complex and the putative functions of these DLCs in vivo, we studied their distributions in adult rat hippocampal formation with the use of light microscopy (LM) and electron microscopy (EM). In this report we show that Tctex-1 and RP3 are localized differentially in hippocampal neurons at both the cellular and the subcellular levels and that these two DLCs are complexed into mutually exclusive subpopulations of dynein. Finally, we have found that Tctex-1 and RP3 are highly enriched at presynaptic and postsyn- 
aptic sites, respectively, suggesting that they may have important roles in synaptic functions.

\section{MATERIALS AND METHODS}

Generation and affinity purification of antibodies. The production of affinity-purified anti-Tctex-1 antibody has been described previously (Tai et al., 1998). This antibody specifically recognizes a single band of $14 \mathrm{kDa}$ on immunoblots of retinal lysates and superior cervical ganglion neurons isolated from rats and does not recognize the closely related DLC-RP3 (Tai et al., 1998; Yano et al., 2001) (also see Results). Anti-RP3 antibody was produced and purified similarly to the anti-Tctex-1 antibody. In brief, bacterially expressed purified glutathione $S$-transferase (GST)-RP3 fusion protein was used as an immunogen for the production of rabbit antiserum (Cocalico, Reamstown, PA). To remove cross-reactive antibodies, we passed the immunized serum sequentially through three CNBr-activated Sepharose CL-4B columns conjugated with Escherichia coli: DH $5 \alpha$ lysate, GST protein, and maltose binding protein (MBP). The final flow-through was affinity purified on an MBP-RP3 Sepharose column, eluted with $0.1 \mathrm{M}$ glycine, $\mathrm{pH} 2.8$, and neutralized with $1 \mathrm{M}$ Tris-Cl, pH 9.5. All GST (Amersham Pharmacia Biotech, Arlington Heights, IL) and MBP (New England Biolabs, Beverly, MA) fusion proteins were produced and purified according to the manufacturers' instructions.

Transfection and immunofluorescent staining of cell cultures. Human embryonic kidney (HEK) 293 cells grown on glass coverslips were transfected with FLAG-Tctex-1 or FLAG-RP3 expression vector by using the calcium phosphate method as described by Sung et al. (1993). At $24 \mathrm{hr}$ after transfection the cells were fixed with methanol, washed in PBS containing $2 \mathrm{mM} \mathrm{MgCl}$ and $0.2 \mathrm{mM} \mathrm{CaCl}_{2}$ (PBS-C/M), blocked with $3 \%$ BSA in PBS-C/M, and then incubated with anti-FLAG antibody (clone M2, 1:500 dilution; Kodak/IBI, New Haven, CT) together with anti-Tctex-1 or with anti-RP3 antibody for $1 \mathrm{hr}$ at room temperature. Then the samples were washed and incubated with Alexa-488-conjugated goat anti-rabbit IgG and Alexa-594-conjugated goat anti-mouse IgG (1:250 dilution; Molecular Probes, Eugene, OR) for visualization. The samples were examined on an Axioskop 2 epifluorescent microscope (Zeiss, Oberkochen, Germany) equipped with the appropriate filter sets, and images were captured by a SPOT 2 cooled CCD camera (Diagnostic Instruments, Sterling Heights, MI).

Immunoprecipitation and immunoblotting of brain extracts. Rat brains except the brainstems and the cerebellums were homogenized in buffered sucrose ( $0.32 \mathrm{~m}$ sucrose, $4 \mathrm{~mm}$ HEPES, $\mathrm{pH}$ 7.3, $1 \mathrm{~mm}$ PMSF, $2 \mu \mathrm{g} / \mathrm{ml}$ aprotinin, $2 \mu \mathrm{g} / \mathrm{ml}$ leupeptin, and $0.7 \mu \mathrm{g} / \mathrm{ml}$ pepstatin) in a glass Teflon homogenizer, using 12 up-and-down strokes at $900 \mathrm{rpm}$. Then the brain homogenate was centrifuged at $800 \times g$ for $10 \mathrm{~min}$ to obtain the postnuclear supernatant. The supernatant was used for the direct immunoblotting assay $(20 \mu \mathrm{g}$ of total protein per lane); before loading, the samples were heated in Laemmli sample buffer and spun at $10,000 \times g$ for $10 \mathrm{~min}$ to remove the aggregation. For the immunoprecipitation experiment a final $1 \%$ Triton X-100 was added to the postnuclear supernatant, and Triton X-100-insoluble materials were removed by centrifugation $(9200 \times g$ for $15 \mathrm{~min})$. These brain detergent lysates then were immunoprecipitated with Tctex-1 or RP3 antibody bound to protein A-Sepharose as described previously (Tai et al., 1998). Brain homogenates or the immunoprecipitates were analyzed on $4-20 \%$ gradient SDS-PAGE (Novex, San Diego, CA), transferred to nitrocellulose, and blotted with Tctex-1 antibody, RP3 antibody, or dynein intermediate chain monoclonal antibody (clone 74.1, Chemicon, Temecula, CA). Immunodetections were performed with the Proto-Blot system (Promega, Madison, WI).

LM localization of Tctex -1 and RP3. All methods involving live animals were approved by the Weill Medical College of Cornell University Institutional Animal Care and Use Committee. Sprague Dawley rats (300-325 gm; Taconic, Germantown, NY) were anesthetized with Nembutal $(150 \mathrm{mg} / \mathrm{kg}$, i.p.) and perfused sequentially through the ascending aorta with (1) normal saline $(0.9 \%)$ containing $1000 \mathrm{U} / \mathrm{ml}$ of heparin (10-15 ml); (2) $50 \mathrm{ml}$ of $3.75 \%$ acrolein (Polysciences, Warrington, PA) and $2 \%$ paraformaldehyde in $0.1 \mathrm{M}$ phosphate buffer $(\mathrm{PB}), \mathrm{pH} 7.4$; and (3) $200 \mathrm{ml}$ of $2 \%$ paraformaldehyde in PB. The brains were removed, and coronal blocks ( $5 \mathrm{~mm}$ thick) were cut and stored in $2 \%$ paraformaldehyde for an additional $30 \mathrm{~min}$. Sections (40 $\mu \mathrm{m}$ thick) were cut on a Vibratome (Leica, Nussloch, Germany), collected in PB, and then placed in $1 \%$ sodium borohydride in PB for 30 min to remove excess aldehydes. The LM immunocytochemical methods were similar to those described previously (Chuang et al., 1999). Briefly, the sections were incubated with anti-Tctex-1 antibody (1:500 dilution) or anti-RP3 antibody (1:500 dilu- tion) in the presence of $0.25 \%$ Triton X-100 and $0.1 \%$ BSA overnight at room temperature and then for $24 \mathrm{hr}$ at $4^{\circ} \mathrm{C}$. Sections were rinsed and incubated with biotinylated goat anti-rabbit IgG (1:400 dilution; Vector Laboratories, Burlingame, CA) for $30 \mathrm{~min}$, followed by avidin-biotin complex (Vector Laboratories) for $30 \mathrm{~min}$ before the diaminobenzidine substrate and $\mathrm{H}_{2} \mathrm{O}_{2}$ color development. The stained sections were mounted on acid-cleaned slides previously coated with $1 \%$ gelatin. The slides were air dried, dehydrated, and coverslipped with DPX (Aldrich Chemical, Milwaukee, WI). The final preparations were examined on a Axioskop 2 microscope equipped with DIC optics. For all of the DLC immunolabeling experiments, adjacent brain sections were used and processed in parallel. Hippocampal nomenclature is consistent with that of Patton and McNaughton (1995).

Immunofluorescent staining of rat hippocampal formation. For immunofluorescent labeling of hippocampal sections, vibratome sections (40 $\mu \mathrm{m}$ thick) were prepared as described above. Rabbit anti-Tctex-1 antibody (1:25 dilution) was incubated with either mouse anti-parvalbumin antibody (1:1500 dilution; Sigma, St. Louis, MO) or goat anti-calretinin antibody (1:2500 dilution; Chemicon), followed by the corresponding Alexa-488- and Alexa-594-conjugated secondary antibodies. For the labeling of TOAD-64 (Turned On After Division), consecutive vibratome sections were collected and labeled with rabbit antibody against Tctex-1 or TOAD-64 (clone Tuc-4, 1:2000 dilution; Chemicon). The immunostaining was examined with either an Axioskop 2 epifluorescent microscope or a laser-scanning confocal microscope (Zeiss).

EM localization of Tctex-1 and RP3. Sections through the hippocampal formation of three rats were processed for the immunoperoxidase localization of Tctex-1 and RP3. For this procedure the sections were prepared as described for LM except that the sections were permeabilized by the "freeze-thaw" technique, and Triton X-100 was omitted in the primary antibody diluent (Milner and Veznedaroglu, 1992). Specifically, brain sections were placed in the cryoprotectant solution $(25 \%$ sucrose and $3 \%$ glycerol in $0.05 \mathrm{M} \mathrm{PB}$ ) for $15 \mathrm{~min}$ before they were submerged into liquid Freon and then in liquid $\mathrm{N}_{2}$. The sections were thawed subsequently in $0.1 \mathrm{M} \mathrm{PB}$ at room temperature and processed for the primary antibody incubation.

Other sections through the hippocampal formation of three rats were prepared for immunogold localization of Tctex-1 and RP3, following the procedures described by Chan et al. (1990). For this experiment the rats were pretreated with a zinc chelator (sodium diethyldithiocarbamate; Fluka, Ronkonkoma, NY) before perfusion with a fixative to diminish the silver background labeling (Veznedaroglu and Milner, 1992). The sections were incubated with sodium borohydride and then permeabilized by the freeze-thaw technique as described above. Next the sections were incubated in Tctex-1 antibody (1:25 dilution) or the RP3 antibody (1:25 dilution) overnight at room temperature and for an additional $24 \mathrm{hr}$ at $4^{\circ} \mathrm{C}$. After several washes the sections were incubated for $2 \mathrm{hr}$ at room temperature with goat anti-rabbit $\mathrm{IgG}$ that was conjugated to $1 \mathrm{~nm}$ gold particles (1:50 dilution; AuroProbe One, Amersham Pharmacia Biotech) in PBS containing $0.1 \%$ gelatin and $0.8 \%$ BSA. Sections were rinsed with PBS, post-fixed in $1.25 \%$ glutaraldehyde in PBS for $10 \mathrm{~min}$, and rinsed once in PBS and once in $0.2 \mathrm{~m}$ sodium citrate buffer, $\mathrm{pH}$ 7.4. Then the conjugated gold particles were enhanced by treatment with silver solution (IntenSE, Amersham Pharmacia Biotech) for 6-8 min. Control for nonspecific silver reactivity included the omission of incubation of the primary antibody or incubation of normal rabbit IgG.

The sections processed for either the immunoperoxidase or immunogold-silver procedures were fixed with $2 \%$ osmium tetroxide for $1 \mathrm{hr}$, dehydrated, and embedded in Epon 812. Ultrathin sections $(50 \mathrm{~nm}$ thick) were prepared and counterstained by $5 \%$ uranyl acetate (Electron Microscopy Sciences, Fort Washington, PA) for 20 min and with Reynolds lead citrate $(2.7 \%)$ for $7 \mathrm{~min}$ before examination on a Philips CM10 electron microscope (Mahwah, NJ). The nomenclature used in the ultrastructural analyses was consistent with that of Peters et al. (1991).

To provide an estimate of the relative proportion of the types of Tctex-1 and RP3-labeled presynaptic and postsynaptic profiles in the dentate gyrus, we performed a semiquantitative EM analysis in a manner similar to that described previously (Barker-Gibb et al., 2001). Briefly, $55 \times 55 \mu \mathrm{m}$ grid squares (each containing a $3025 \mu \mathrm{m}^{2}$ field) from the central hilar region adjacent to the plastic tissue interface of one block each, labeled for either Tctex-1 or RP3, were selected for analysis. The grid squares were selected randomly and were located at a depth with no apparent visible nonspecific staining (i.e., edge artifact). The localizations of Tctex-1 or RP3 immunoreactivity near synapses were catego- 

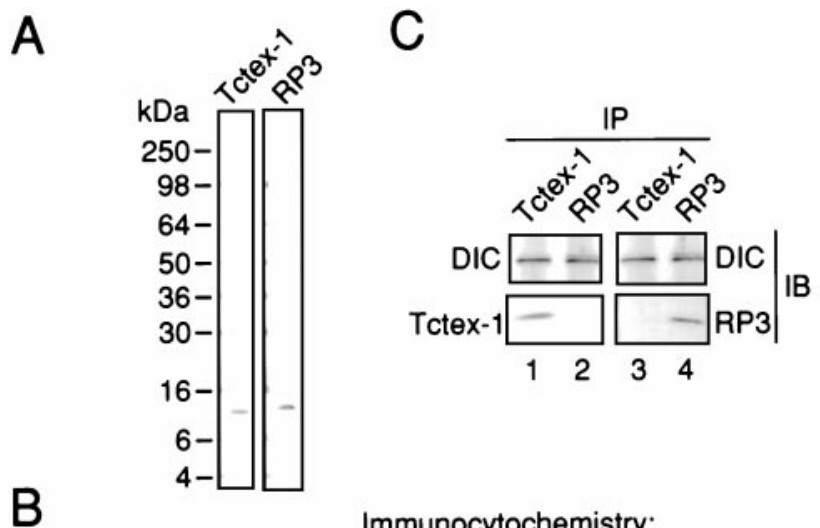

Immunocytochemistry:

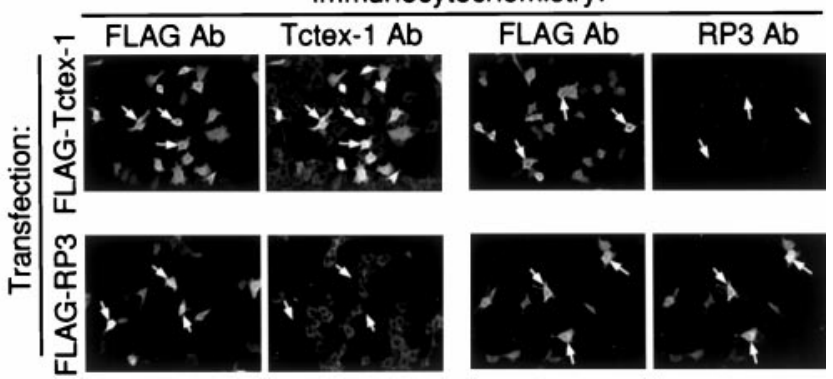

Figure 1. Specific detection of Tctex-1 and RP3 in rat brain lysate, transfected cells, and immunoprecipitates and assembly of Tctex-1 and RP3 in distinct dynein complexes. $A$, Rat brain lysate was resolved on SDS-PAGE and immunoblotted with anti-Tctex-1 or anti-RP3 antibody. Both antibodies recognized a single $\sim 14 \mathrm{kDa}$ molecule, although Tctex-1 migrated slightly faster than RP3 on SDS-PAGE. B, HEK 293 cells transfected with FLAG-Tctex-1 or FLAG-RP3 expression vector were double labeled with anti-FLAG monoclonal antibody and anti-Tctex-1 or anti-RP3 antibody (as indicated). Although the anti-FLAG antibody recognized both transfected FLAG-Tctex-1 and FLAG-RP3, Tctex-1 antibody recognized only FLAG-Tctex-1, but not FLAG-RP3. Arrows point to examples of transfected cells. Likewise, anti-RP3 antibody immunolabeled only the FLAG-RP3, but not FLAG-Tctex-1, transfected cells. Note that anti-Tctex-1 antibody also lightly labeled the endogenous Tctex-1 present in the nontransfected cells (left panels). There is no detectable endogenous RP3 in HEK 293 cells. $C$, Cytoplasmic dynein complexes were immunoprecipitated from brain lysates with anti-Tctex-1 or anti-RP3 antibody. The immunoprecipitates were separated on SDSPAGE and transferred to nitrocellulose. The top half of the filter was immunoblotted with anti-dynein intermediate chain $(D I C)$ to demonstrate that approximately equal amounts of the dynein complex were precipitated by each antibody (top panel). The bottom half of the nitrocellulose filter was immunoblotted with anti-Tctex-1 or anti-RP3 antibody. Anti-Tctex-1 antibody detected Tctex- 1 in the anti-Tctex- 1 precipitate, but not in the anti-RP3 precipitate. Likewise, anti-RP3 detected RP3 in the anti-RP3 precipitate, but not in the anti-Tctex-1 precipitate. This result suggests that the dynein complex precipitated by Tctex-1 antibody did not contain RP3, and vice versa. Furthermore, Tctex-1 antibody and RP3 antibody did not cross-react with the antigens of each other.

rized with regard to presynaptic or postsynaptic localization and tabulated.

\section{RESULTS}

\section{Specificity of Tctex-1 and RP3 antibodies}

Two affinity-purified antibodies were produced to examine the assembly of Tctex-1 and RP3 in dynein complex and immunolocalization in vivo. Several complementary methods confirmed the specificity of these antibodies. First, both anti-Tctex-1 and antiRP3 antibodies recognized a single $14 \mathrm{kDa}$ band in rat brain lysates in immunoblots (Fig. $1 A$ ). Second, these two antibodies recognized their own antigen, but not the antigen of each other, when they were immunoblotted for the corresponding bacterial fusion proteins (data not shown). Finally, although anti-FLAG antibody recognized both FLAG-Tctex-1 and FLAG-RP3 in transiently transfected HEK 293 cells, anti-Tctex-1 and anti-RP3 antibodies could recognize specifically only the FLAG-Tctex-1 and the FLAG-RP3, respectively (Fig. 1B). Because HEK 293 cells themselves expressed endogenous Tctex-1, but not RP3 (J.-Z. Chuang and C.-H. Sung, unpublished observation), a low level of endogenous Tctex-1 labeling also could be detected by anti-Tctex-1 antibody in nontransfected cells.

\section{Presence of Tctex-1 and RP3 in different dynein complexes}

Each cytoplasmic dynein complex contains two $14 \mathrm{kDa}$ DLCs (King et al., 1998); however, whether one Tctex-1 and one RP3 or two of the same isoform are assembled in the same dynein complex is unknown. To help clarify this issue, we determined whether the cytoplasmic dynein complexes immunoprecipitated from brain lysates by anti-Tctex- 1 contained only Tctex- 1 or both DLCs and, conversely, whether dynein immunoprecipitated by anti-RP3 contained only RP3 or both DLCs. In these experiments the dynein intermediate chain was present in both antiTctex-1 and anti-RP3 immunoprecipitates (Fig. 1C, top panel), demonstrating that both anti-Tctex-1 and anti-RP3 antibodies could precipitate the dynein complex effectively. However, only Tctex-1, but not RP3, was detected in the anti-Tctex-1 immunoprecipitates (Fig. 1C, bottom panel). Conversely, only RP3, but not Tctex-1, was detected in the anti-RP3 immunoprecipitates. These results suggest that, at least in brain, alternative $14 \mathrm{kDa}$ DLCs are assembled in mutually exclusive dynein complexes. Furthermore, Tctex-1 immunoprecipitated from rat brain extracts was recognized by anti-Tctex-1 antibody, but not by anti-RP3 antibody, in immunoblots and vice versa, adding further evidence that anti-Tctex-1 and anti-RP3 antibodies do not cross-react with each other.

We and others have shown that Tctex-1 and RP3 bind directly to the dynein intermediate chain (Mok et al., 2001; Tai et al., 2001). The capability of anti-Tctex- 1 and anti-RP3 antibodies to immunoprecipitate intermediate chain suggested that these antibodies did not disrupt the respective light chain-intermediate chain interaction. It further suggested that the epitopes of these antibodies are unlikely to be buried in the interface of the protein-protein interaction. Although the possibility cannot be excluded, epitope masking is unlikely to be a concern for such antibodies in the following immunolocalization studies.

\section{Differential distribution of Tctex-1 and RP3 in rat hippocampal formation and cerebral cortex}

To reveal whether the dynein complexes containing Tctex- 1 and RP3 were distributed differentially in vivo, we examined the topographic localization of these two DLCs in the rat brain. LM localization of Tctex-1 and RP3 immunoreactivities in coronal sections of the adult rat brain revealed that the general distributions of these two molecules were markedly different and that the difference was particularly obvious in the hippocampal formation and the cerebral cortex (Fig. 2). In the hippocampal formation the most intense Tctex-1 labeling was associated with perikarya of a single layer of cells located at the subgranular zone of the dentate gyrus (Fig. 2A,B). The labeled dendritic processes derived from these cells often penetrated the granule cell layer. Tctex-1 labeled perikarya also were distributed sporadically throughout the remainder of the hippocampal formation. A moderate level of 

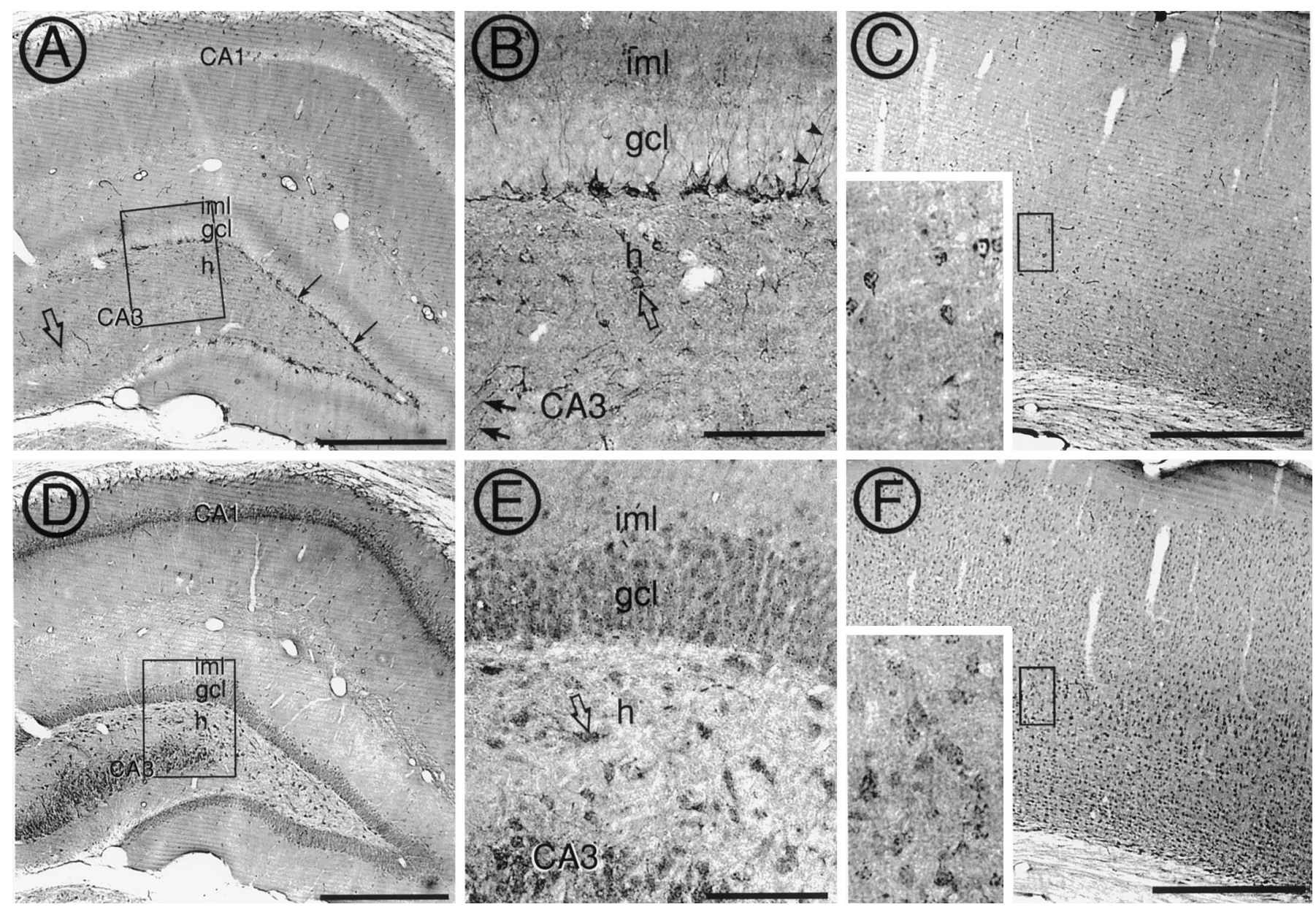

Figure 2. Distinct distributions of RP3 and Tctex-1 in the hippocampal formation and cerebral cortex. Coronal sections of adult Sprague Dawley rat brain were immunolabeled for Tctex-1 $(A-C)$ or RP3 $(D-F)$, using affinity-purified antibodies, followed by peroxidase color development with diaminobenzidine. $A$, Perikarya exhibiting intense Tctex-1 immunoreactivity were found in the subgranular zone (arrows) of the dentate gyrus. Neurons containing Tctex-1 labeling also were scattered throughout the entire hippocampal formation. Moderate levels of diff usive Tctex-1 labeling were found in the inner molecular layer $(\mathrm{iml})$ and hilus of the dentate gyrus $(h)$ and stratum lucidum of CA3 region (open arrow). B, Higher magnification of the boxed area in $A$ shows that dendritic processes of Tctex-1 labeled somata in the subgranular zone often penetrated (arrowheads) through the granule cell layer $(g c l)$. Both Tctex-1-labeled perikarya (open arrow) and varicose processes (arrows) were found throughout the hilus and CA3 regions. $C$, A low-magnification view of the cerebral cortex (layers 1-6) shows Tctex-1-immunoreactive neurons scattered throughout all layers. A higher magnification micrograph of the boxed area in layer 5 (inset) revealed perikaryal labeling of Tctex-1 in a small subset of pyramidal cells in cerebral cortex. In addition, many varicose processes were also Tctex-1-immunoreactive. $D$, Diff use RP3 immunoreactivity was concentrated in the perikarya of the CA1 and CA3 pyramidal cells and granule cells. $E$, A higher magnification micrograph of the boxed area in $D$ demonstrates that RP 3 immunoreactivity also was found in many small puncta over the granule cell layer $(\mathrm{gcl})$, hilar interneurons (arrow), and CA3 neurons. $F$, The majority of cerebral cortical neurons was RP3-immunoreactive. The inset reveals the diffuse as well as the grainy perikarya labeling of RP3 in these pyramidal cortical neurons. Scale bars: $A$, $C$, $D, F, 500 \mu \mathrm{m} ; B, E, 100 \mu \mathrm{m}$.

diff use Tctex-1 labeling was found in the inner molecular layer of the dentate gyrus (Fig. $2 A$ ) and in stratum lucidum of CA3 region (Fig. $2 A$, open arrow). In addition, in the dentate hilus many fine varicose processes and interneuron-like somata were also Tctex1-immunoreactive (see below). Finally, a low level of Tctex-1 immunoreactivity was present throughout the remainder of the hippocampal formation, with the exception of the somata of pyramidal and granule cells, which were almost completely unstained.

The pattern of RP3 immunoreactivity in the hippocampal formation was remarkably different from Tctex-1 immunoreactivity. In particular, the perikarya of the principal neurons in the pyramidal cell and granule cell layers and interneurons in the dentate hilus contained diffuse RP3 immunoreactivity throughout their cytoplasm (Fig. 2D,E). In addition, intense puncta of RP3 immunoreactivity were found over principal cells and in the hilus of the dentate gyrus. The distinct and almost complementary staining patterns of Tctex-1 and RP3 suggested that these two DLCs are expressed in different subsets of hippocampal neurons and/or different subcellular compartments within certain neurons.

In agreement with the observations in the hippocampal formation, Tctex-1 and RP3 antibodies also recognized very distinct populations of cerebral cortical neurons (Fig. 2C,F). Throughout all layers of cerebral cortex the majority of pyramidal cells was RP3-immunoreactive. Diff use perikarya and intense puncta labeling were seen in these cells. In contrast, many fewer Tctex-1positive pyramidal cells were scattered throughout the cortex, and the immunoreactivity was concentrated in the perinuclear region as well as in the varicose fiber. As seen in the higher magnification of layer 5 cerebral cortex (Fig. 2C,F, insets), Tctex-1- and RP3immunoreactive neurons were mainly nonoverlapping, suggesting different subsets of cortical neurons contained different DLCs. 

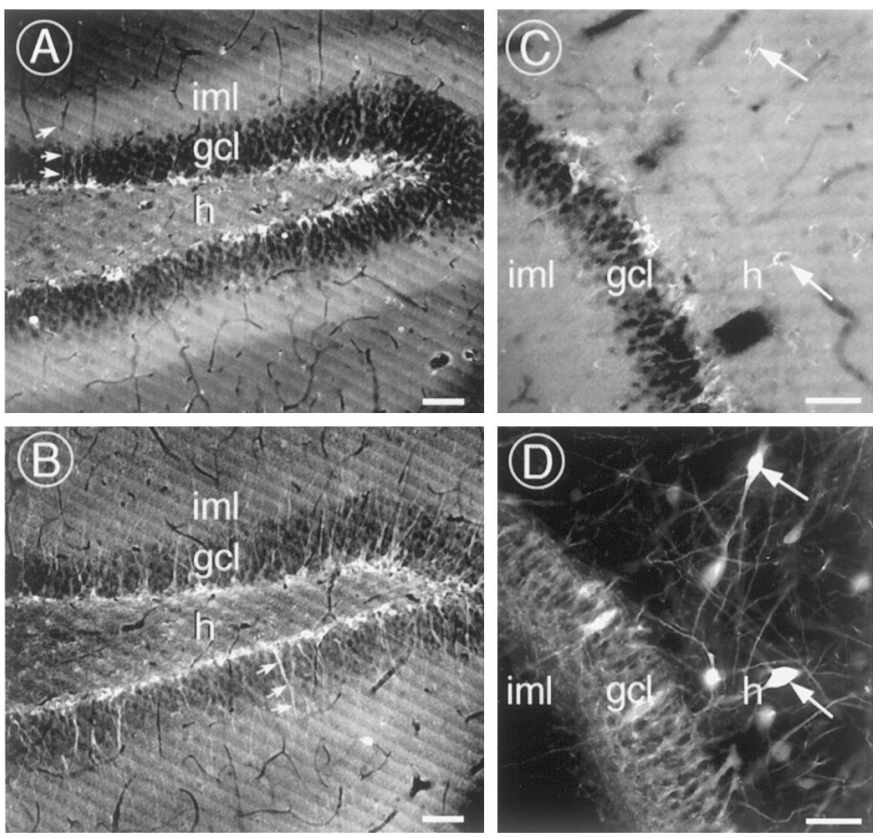

Figure 3. Fluorescent micrographs of Tctex-1 immunoreactivity in TOAD-64-containing neurons and a subset of parvalbumin-containing interneurons. $A, B$, Consecutive coronal sections of the rat brains were singly labeled with either Tctex-1 $(A)$ or TOAD-64 $(B)$ antibodies, followed by Alexa-488-conjugated goat anti-rabbit IgG. Neurons labeled for Tctex-1 $(A)$ had an almost identical distribution to neurons labeled with TOAD-64 $(B)$ in the subgranular zone. The dendritic processes (arrows) of Tctex-1 and TOAD-64 perikarya penetrated through the granule cell layer. $C, D$, Confocal images of hippocampal slices were double labeled with Tctex-1 rabbit antibody $(C)$ and parvalbumin monoclonal antibody $(D)$, followed by corresponding secondary antibodies. Tctex-1 labeling sometimes colocalized with parvalbumin-labeled cells (arrows) in the hilus of the dentate gyrus. Scale bars, $50 \mu \mathrm{m}$.

\section{Tctex-1 immunoreactivity is found in newly born neurons and a subset of interneurons}

The LM staining of Tctex-1 in the hippocampal formation highlighted a group of cells that formed a nearly continuous single layer along the granule cell/hilus border (Fig. 2B). This region has been described as a region of postnatal neurogenesis (Altman and Das, 1965; Kaplan and Hinds, 1977; Bayer and Yackel, 1982; Gueneau et al., 1982; Cameron et al., 1993; Scott et al., 1998). In this subgranular zone a local population of progenitor cells generates new granule cells. These postmitotic progeny migrate into the granule cell layer and begin differentiation by extending their processes to target cells. To determine whether Tctex- 1 immunoreactivity was associated with newborn neurons, we compared the staining patterns of Tctex-1 and the postmitotic neuron-specific marker TOAD-64 (Minturn et al., 1995a,b) in adjacent brain sections. As described above, Tctex-1 immunoreactivity was concentrated in the subgranular zone of the dentate gyrus (Fig. $3 A$ ). As expected (Parent et al., 1997; Scott et al., 1998; Wang et al., 2000), TOAD-64 immunoreactivity also was confined to the cell bodies and processes of cells in the same region (Fig. 3B). Although we were not able to double-label Tctex-1 and TOAD-64 in the same section because both antibodies were generated in rabbits, the similar and extensively overlapping localizations in the subgranular cell layer suggest that Tctex-1 is highly enriched in newly generated neurons in adult animals. Subgranular Tctex-1-positive cells also were colocalized with a neuron-specific marker, $\beta$ III tubulin (data not shown), further confirming their

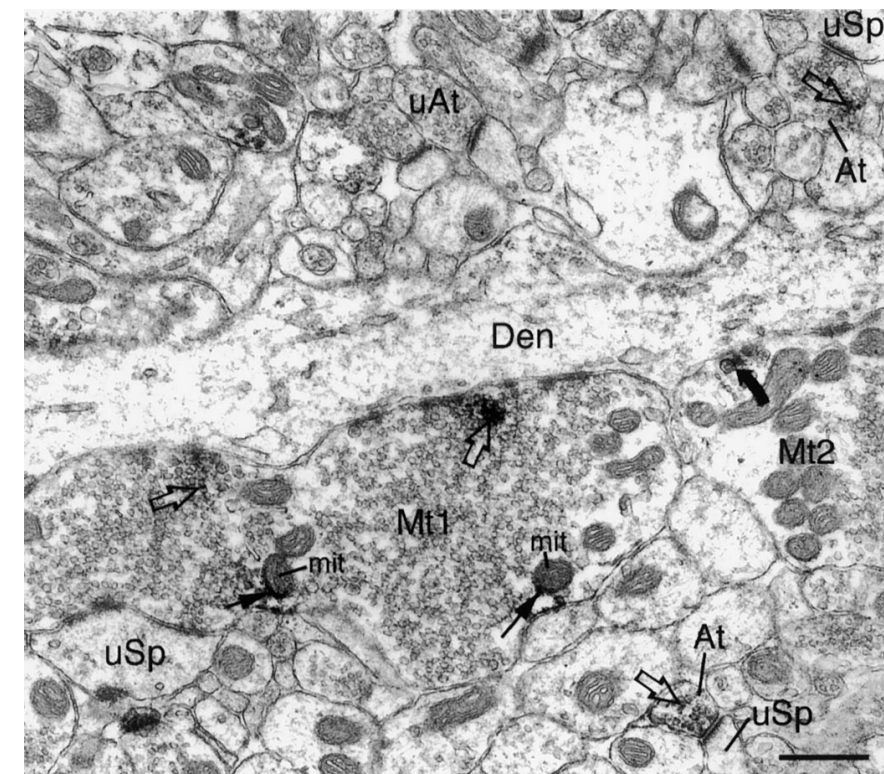

Figure 4. Electron micrograph of the immunoperoxidase labeling of Tctex-1 in the central hilus. Patches of Tctex-1 immunoprecipitate were associated primarily with the small synaptic vesicle clusters (open arrows) and mitochondria (mit; arrows) in a mossy fiber terminal (Mt) and small axon terminals $(A t)$. In one mossy fiber terminal (Mt1) some intensely labeled synaptic vesicles were closely apposed to the synaptic specialization. Near the synaptic contact of mossy fiber terminal $2(M t 2)$ a large pleomorphic vesicle also had Tctex-1 immunoreaction products affiliated with it (curved arrow). Almost all of the spines contacted by Tctex-1positive terminals are unlabeled. USp, Unlabeled spine; UAt, unlabeled axon terminal. Scale bars, $0.5 \mu \mathrm{m}$.

neuronal identity. In contrast, a high level of RP3 (Fig. 2E), but a relatively low level of Tctex-1 (Fig. $2 B$ ), was seen in the cell bodies of mature dentate granule cells.

Because the perikaryal labeling of Tctex- 1 in the dentate hilar area (Figs. $2 A, 3 A$ ) also was similar to GABAergic interneurons containing parvalbumin or calretinin (Seress et al., 1993; Mikkonen et al., 1997), the sections were double labeled with anti-Tctex-1 antibody and these two interneuron markers. No Tctex-1 labeling was found on calretinin-positive interneurons (data not shown). However, a subset of parvalbumin-positive interneurons was immunoreactive for Tctex-1 (Fig. 3C,D). The selective presence of Tctex-1 in a subpopulation of parvalbuminpositive interneurons further suggests that Tctex-1 localization may be regulated tightly.

\section{Membrane organelle and axon terminal association of Tctex-1}

To define and compare the distribution of these two DLCs at the subcellular level, we examined the Tctex-1 and RP3 immunoreactivities by the pre-embedding EM technique. For this procedure the dentate hilar region near the CA3 subfield was chosen for analysis because significant levels of both Tctex-1 and RP3 immunoreactivities were detected in this region by LM.

When the ultrastructural localization of Tctex-1 was examined by the immunoperoxidase method in the central hilus, in addition to somata in the subgranular hilus, prominent Tctex-1 labeling was associated with clusters of the small synaptic vesicles in axon terminals (Fig. 4). Notably, these clusters of synaptic vesicles labeled with Tctex-1 often were affiliated with synaptic contacts. Occasionally, larger vesicles with amorphous internal membranes near the dendritic synapses were also Tctex-1-immunoreactive 
(Fig. 4, curved arrow). In contrast, most of the dendritic spines and the postsynaptic elements on dendrite profiles lacked significant Tctex-1 immunoprecipitate. In the semiquantitative analysis of the central hilus, $95 \%$ (of 236) of the Tctex-1-immunoreactive synaptic profiles were classified as axon varicosities and axon terminals. The Tctex-1 immunoperoxidase precipitate was rarely distributed homogeneously, suggesting that these immunolabeled DLCs are not free subunits, which otherwise would be cytosolic.

The immunogold labeling method, which is less sensitive than the immunoperoxidase method but provides better resolution, also was used to examine the organelle association of Tctex-1. Consistent with the immunoperoxidase labeling, the highest density of Tctex-1 immunogold was found to be affiliated with the axon terminals (Fig. 5A-D). Many of these Tctex-1-positive terminals had the morphological characteristics of mossy fiber: they were large $(>1.0 \mu \mathrm{m})$, filled with small synaptic vesicles (Fig. 5 $C, D)$ (Amaral, 1978; Commons and Milner, 1995). However, Tctex-1-immunoreactive small axon terminals also were detected and often contacted dendritic spines (Fig. 5A,C). Within both types of terminals, Tctex-1-derived gold particles were associated with a subset of synaptic vesicles predominantly, but not exclusively, near the active zone. Interestingly, some Tctex-1associated vesicles in the axon terminals were pleomorphic and slightly larger than the uniform small synaptic vesicles typical of the mature active zone ( $80-100$ vs $\sim 40 \mathrm{~nm}$ in diameter). Although some of these Tctex-1-immunoreactive vesicles might have been large dense-core vesicles, most appeared to be translucent. Similar presynaptic Tctex-1 labeling also was observed in the inner molecular layer (Fig. 5C), suggesting that Tctex-1 may have a prominent role in presynaptic profiles. Tctex-1 labeling occasionally was associated with smooth endoplasmic reticulum in both types of axon terminals (Fig. $5 B, C$ ). In contrast, no significant Tctex-1 immunoreactivity was affiliated with dendritic spines in either the dentate hilus region or molecular layer (Fig. $5 A-D)$. The immunogold labeling in presynaptic profiles was not observed when either the primary antibody was omitted or when sections were incubated with control $\mathrm{IgG}$ (data not shown).

A low density of Tctex-1-derived gold particles also was detected on various membrane structures (e.g., multivesicular bodies, mitochondria, tubulovesicular smooth endoplasmic reticulum) in the axons (Fig. $5 C$ ) and the dendritic shafts (Fig. 5A) of neurons in the hilus. The observation that Tctex-1 immunoreactivity in neurites often was associated with the membranes affiliated with MTs or the MTs themselves (Fig. $5 A$ ) supports the role of dynein in MT-based organelle transport. Moreover, the Tctex1-positive membranes/organelles are likely to represent the cargoes attached to Tctex-1 or to Tctex-1 containing dynein complex. Finally, consistent with the LM results, very little Tctex-1 immunoreactivity was seen in the somata of granule cells, whereas many organelles (e.g., mitochondria, multivesicular body, Golgi apparatus) were labeled in the subgranule cell bodies (data not shown).

\section{The perikaryon and dendritic localization of RP3}

The ultrastructural localization of RP3 also was examined by both the immunoperoxidase and the immunogold methods. Consistent with the LM results, the immunoperoxidase method revealed that the RP3 immunoreactivity was prominent in the perikarya of the granule cells. Specifically, RP3 labeling was associated primarily with the Golgi apparatus (Fig. 6A,B). In the Golgi, RP3 immunoreactivity was found in the membrane stacks as well as at the slightly dilated ends. On the trans (concave) face of the Golgi

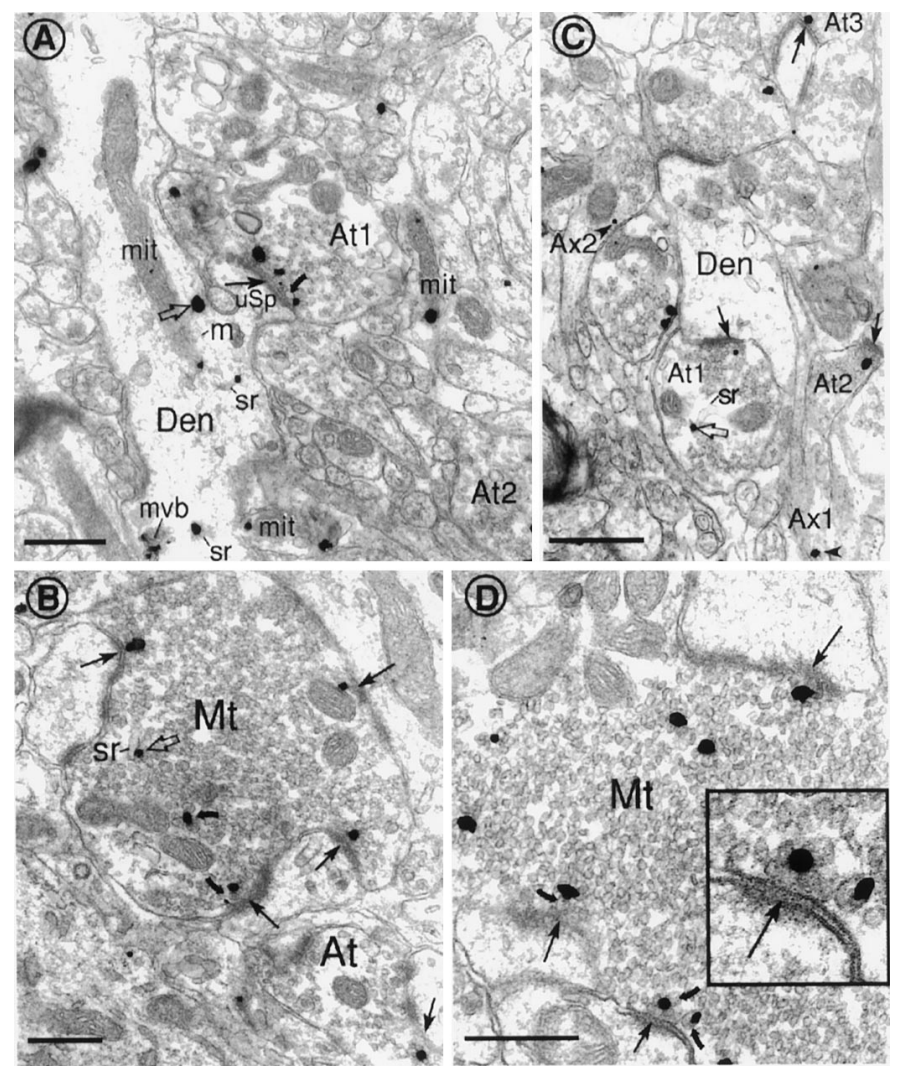

Figure 5. Electron micrographs of Tctex-1 immunogold labeling in the hilus $(A, B, D)$ and in the inner molecular layer $(C)$ of the dentate gyrus. $A$, A Tctex-1-labeled axon terminal (At) synapsed on an unlabeled dendritic spine $(u S p)$, emanating from the shaft of an labeled dendrite (Den). In axon terminals, immunogold-silver particles indicative of Tctex-1 were associated with small synaptic vesicles and larger pleomorphic vesicles (curved arrow). These organelles often were affiliated with the active zone (filled arrow). In the dendritic shaft, Tctex-1 immunoreactivity (open arrow) was affiliated with MTs $(m)$, smooth endoplasmic reticulum ( $s r)$, mitochondria (mit), and multivesicular bodies $(m v b)$. B, A large Tctex-1labeled mossy fiber terminal forms multiple asymmetric synapses on dendritic spines that invaginate or indent the terminal. In the labeled terminal, Tctex-1-derived immunogold particles often were found in vesicles that were larger than small synaptic vesicles (curved arrows) and that often were found in the vicinity of the active zone (arrows). Occasionally, Tctex-1 immunogold was seen on smooth endoplasmic reticulum ( $s r)$. $C$, Three small axon terminals (At1, At2, At3) containing Tctex-1 labeling that is affiliated with synaptic vesicles near active zones (arrows) are shown. Tctex-1 immunoreactivity also was associated with smooth endoplasmic reticulum (sr; open arrow) and various membrane profiles (arrowheads) in the neighboring axon $(A x)$. $D$, Immunogold particles indicative of Tctex-1 were associated with synaptic vesicles. Some Tctex-1immunoreactive synaptic vesicles were larger than the small synaptic vesicles (curved arrows) and were located in the vicinity of the active zone (arrows), which is enlarged in the inset. Scale bars, $0.5 \mu \mathrm{m}$.

apparatus, RP3 immunoprecipitation was concentrated with vacuolar/tubular structures, likely to be the membranes budded from the trans-Golgi saccules [also often referred to as the trans-Golgi network (TGN); Peters et al., 1991]. This patched RP3 labeling was intense, large in diameter (average $0.5 \mu \mathrm{m}$ ), and likely to contribute, at least in part, to the labeled puncta seen under the LM. Similarly, immunogold labeling also revealed that RP3 immunolabeling often was affiliated with the Golgi apparatus and its derived tubular vesicles (Fig. 6C). The Golgi and TGN localizations of RP3 support the protein trafficking role of RP3 or RP3-mediated dynein activity, because membrane proteins and 


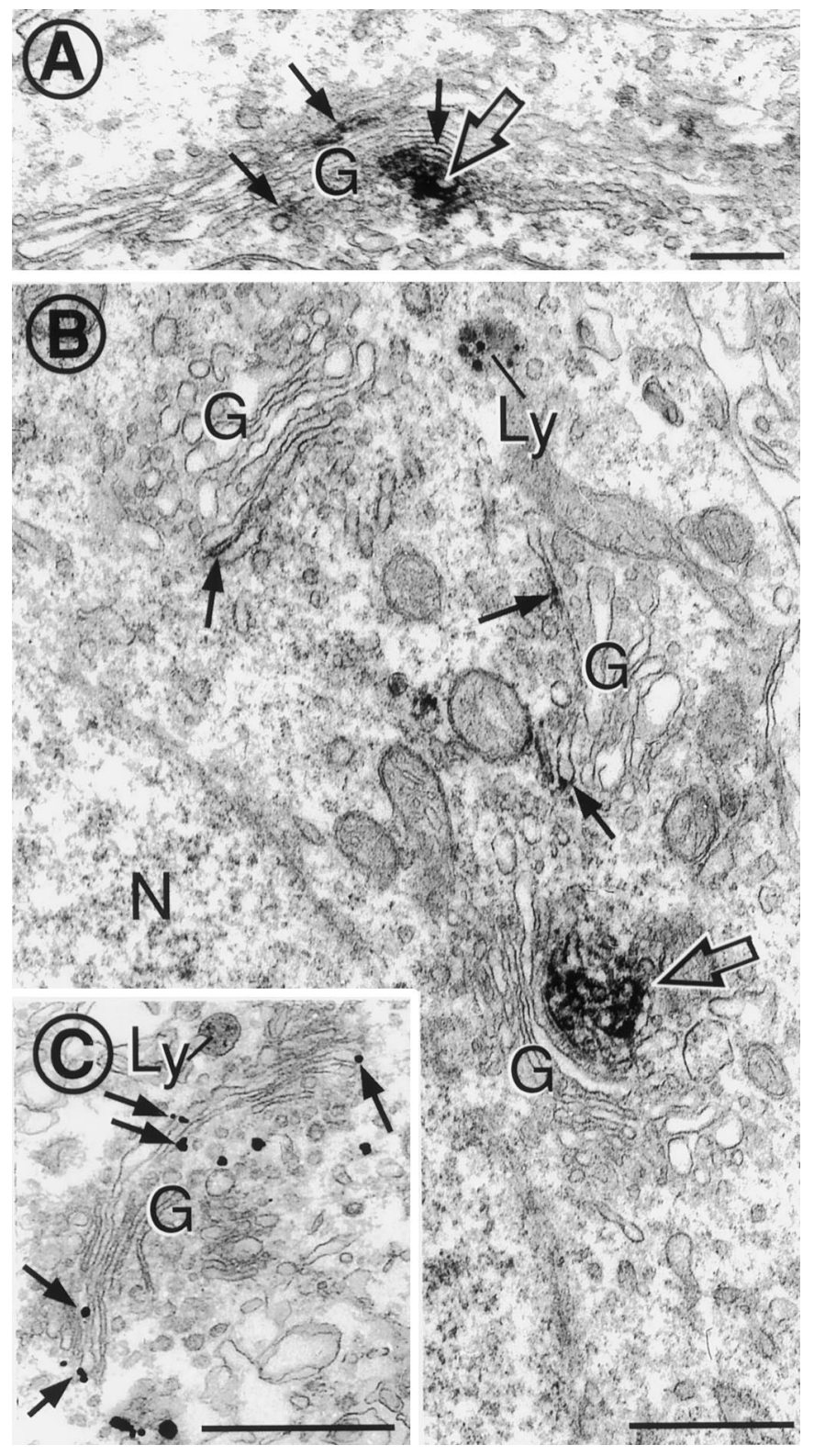

Figure 6. The Golgi localization of RP3 in the granule cells. $A$, In a granule cell perikaryon RP3 immunoreactivity was associated with Golgi cisternae (arrows) and clusters of vesicles likely to be in the trans-Golgi network (open arrow). B, RP3-derived immunoperoxidase precipitate was associated with the Golgi apparatus $(G)$ near the membrane stacks (arrows) as well as the slightly dilated ends. Prominent patches of RP3 immunoreactivity were found in the clusters of vacuolar/tubular membrane profiles on the trans (concave) face of the Golgi complex (open arrow). An unlabeled lysosome ( $L y)$, which contains multiple electrondense grains, also was found. $C$, High density of RP3 immunogold particles was observed on the Golgi membrane stacks and the tubular vesicles on the trans side of the Golgi apparatus. Scale bars, $0.5 \mu \mathrm{m}$.

other proteins in the secretory pathway are thought to be sorted at the TGN before they are translocated further to the destined membrane domains (Nelson, 1992).

Patches of RP3 immunolabeling frequently were associated with a function-unidentified, electron-dense nematosome-like cytoplasmic inclusion (average, $0.7 \mu \mathrm{m}$; data not shown). These cytoplasmic inclusions also could account for the puncta observed under LM. Moreover, RP3 immunoreaction product sometimes was found on multivesicular bodies and mitochondria, whereas

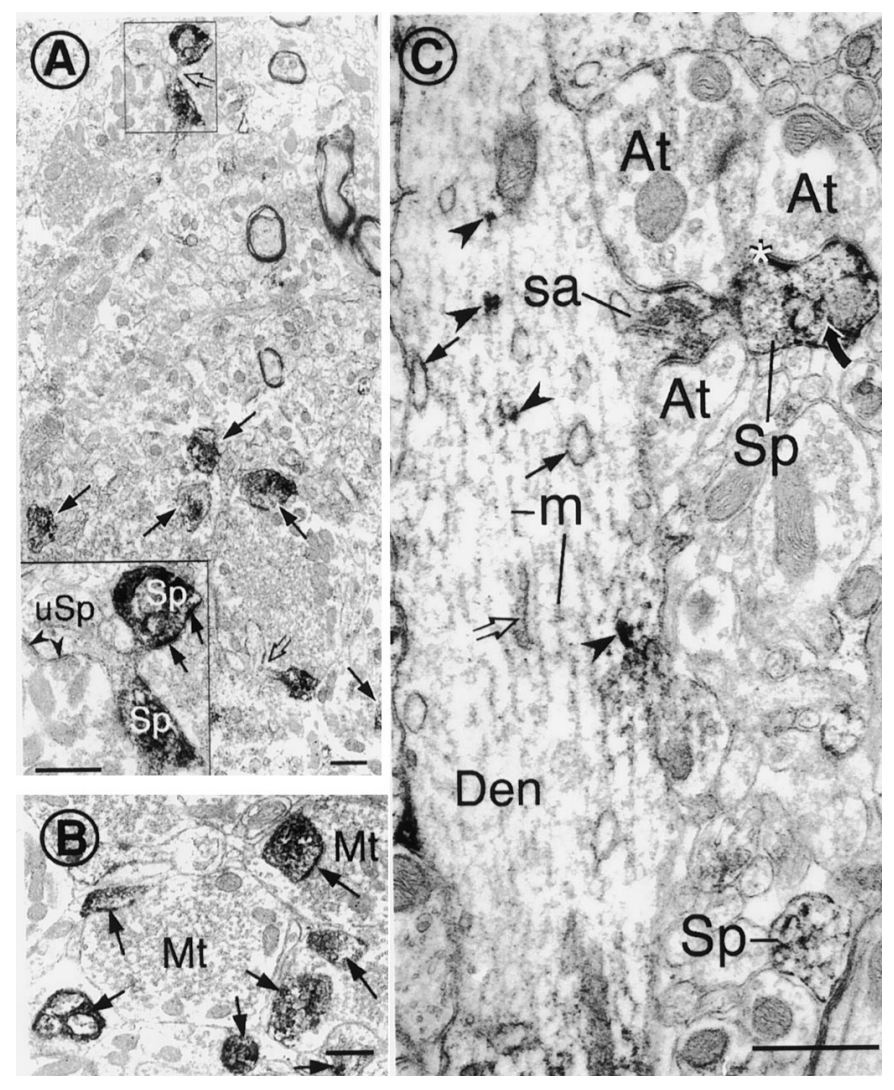

Figure 7. Electron micrographs of the RP3 localization in the hilus proper of the dentate gyrus. $A$, A low-magnification electron micrograph shows that RP3 immunoperoxidase precipitate was found primarily in dendritic spines (filled arrows). RP3-immunoreactive spines frequently originated from the same branched protuberance (open arrows). The inset shows a high-magnification view of a branched spine in which RP3 was present on only two spine heads and absent from another. USp, Unlabeled spine. The PSDs (small arrows) usually were labeled heavily, in contrast to the corresponding PSDs (arrowheads) in an unlabeled spine. B, Several RP3-containing dendritic spines (arrows) invaginated into a few large mossy fiber terminals $(M t)$, which essentially have no reaction products. $C$, In a longitudinally sectioned dendrite (Den) a spine extends and branches into two spine heads, one RP3-positive and one RP3-negative (the branching is marked with an asterisk). The classic spine apparatus ( $s a$ ), which has flattened sacs of smooth endoplasmic reticulum, was decorated by RP3 immunoreactivity. Moreover, intense RP3 labeling was seen routinely on PSDs and surrounding the multivesicular body (curved arrow) within the labeled spine head. In the dendritic shaft, RP3-derived immunoperoxidase precipitate was associated with tubulovesicular smooth endoplasmic reticulum (open arrow) and vesicles of various sizes (arrows) that often were arrayed on MTs $(m)$. Patches of RP3 immunoprecipitates (arrowheads) sometimes were associated closely with the MTs themselves. Scale bars, $0.5 \mu \mathrm{m}$.

RP3 labeling with lysosomes (Fig. 6B) and rough endoplasmic reticula was not observed.

\section{RP3 is highly enriched in postsynaptic profiles}

In the central hilus region the most profound RP3 immunolabeling, as visualized with both the immunoperoxidase and immunogold methods, was affiliated primarily with the dendritic spines and the postsynaptic elements in dendritic shafts that were contacted by axon terminals (Figs. 7-9). The dendritic spine labeling of RP3 was remarkably intense, especially when the immunoperoxidase labeling method was used. In contrast to Tctex-1, RP3 immunoreactivity rarely was seen in the axon terminals. Semiquantitative analysis of the central hilus revealed that $87 \%$ (of 

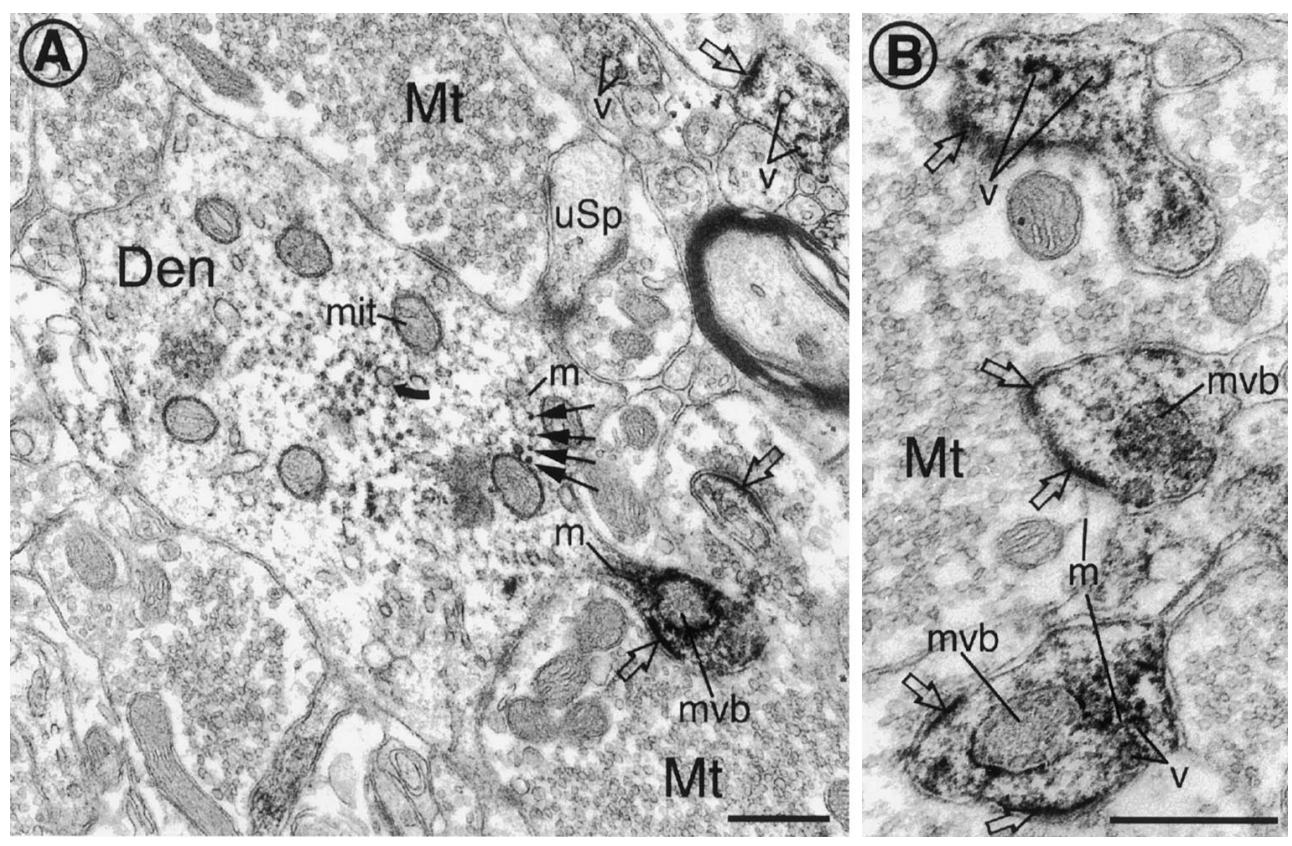

Figure 8. MT and membrane organelle association of RP3 in the postsynaptic elements. $A, \mathrm{RP} 3$ immunoperoxidase precipitate was concentrated near MT $(m)$ tracks (arrows) within a dendritic profile. The outer membranes of mitochondria (mit) and vesicular profiles (curved arrow) within the dendrite also were RP3-immunoreactive. RP3 immunoreactivity was notably enriched in dendritic spines, as demonstrated by the high-level RP3 immunoreaction product associated with membranes of vesicles $(v)$, multivesicular body $(m v b)$, and PSD (open arrow) within spines. No RP3 immunoreactivity was observed in several mossy fiber terminals that were in direct contact with RP3-positive dendrites/spines. B, A high-magnification view of three RP3-positive spine heads invaginated into an RP3-negative mossy fiber terminal. The cytosolic face of multiple spine organelles/structures, such as multivesicular body $(m v b)$, large vesicles $(V)$, and PSD (open arrows), was decorated by the RP3 immunoreaction product. Scale bars, $0.5 \mu$ m.

173) of the RP3-immunolabeled profiles were dendritic spines and postsynaptic sites of shaft synapses.

Many RP3-immunoreactive dendritic spines were highly branched and were contacted by mossy fiber terminals (Fig. 7A$C)$. Based on their topography and their asymmetric synaptic contacts with mossy fiber boutons, at least a fraction of these RP3-immunoreactive spines in the central hilus likely were derived from mossy cells. Mossy cells are one of the most distinctive and common cell type in dentate hilus (Ribak et al., 1985; Frotscher et al., 1991). Moreover, RP3 immunoreactivity frequently was distributed differentially on different spine heads within the same branched spine (Fig. 7A,C).

Immunogold labeling also revealed the predominant postsynaptic labeling of RP3 in spines and in dendritic profiles (Fig. 9). Very often, clusters of immunogold particles appeared on the postsynaptic elements that were contacted by unlabeled axon terminals.

Within the spine head, RP3 immunoreaction product decorated many membrane structures typically seen in the postsynaptic elements, including small smooth vesicles, multivesicular bodies, and the spine apparatus (Figs. 7C, 8, 9). Finally, intense RP3 immunoreactivity also was associated with the postsynaptic density (PSD) in all positive spines (Figs. 7-9), whereas it was absent from the opposing presynaptic active zone. The highly enriched RP3 localization in spines suggests that RP3 is involved functionally in the formation and/or turnover of synaptic membranes and/or synaptic proteins in dendritic spines.

RP3 immunoreactivity also was found in dendrites on multiple membranous structures, including smooth endoplasmic reticulum (Figs. $7 C, 9 A, C$ ), vesicles of various sizes (Figs. $7 C, 8 A, 9 A$ ), and mitochondria (Fig. $8 A$ ). Fine RP3 immunoprecipitate also was affiliated with MTs themselves (Figs. $7 C, 8 A$ ). The close associ- ation between RP3 and MTs or the organelles affiliated with the MTs suggested that these DLC immunoreactivities represent the DLCs in the dynein complex. Finally, substantially lower RP3 immunoreactivity was observed occasionally on membrane structures in the axons (data not shown).

\section{Differential distribution of Tctex-1 and RP3 within granule cells}

The differential synaptic localizations of Tctex-1 and RP3 described above in the hilus region were derived from different cell types, e.g., granule cells and mossy cells, respectively. However, our results also support the notion that both DLCs could coexist in the same cell type and that they are distributed differentially in distinct compartments within it. This argument is based on the observation that in granule cells RP3, but not Tctex-1, is enriched at the somata (Fig. 6) and postsynaptic elements of apical dendrites (data not shown). In contrast, Tctex-1, but not RP3, is enriched at the axon terminals of granule cells (i.e., mossy fiber bouton) (Figs. 4, 5, 7, 8).

Our immunoprecipitation experiments suggested that Tctex-1 and RP3 are not present in the same dynein complex (Fig. 1C). However, at present time we were not able to determine whether Tctex-1 and RP3 or their associated dynein complexes could be simultaneously present on any of those immunoreactive organelles in axon/dendrites of the cells expressing both DLCs, because both anti-Tctex-1 and anti-RP3 antibodies were generated in rabbits. Nevertheless, the common localization of the Tctex-1 and RP3 in the membrane organelles (e.g., smooth endoplasmic reticulum, Golgi apparatus, mitochondria) as well as their unique localization at synapses suggests that dynein complexes with different compositions may possess the ability to 


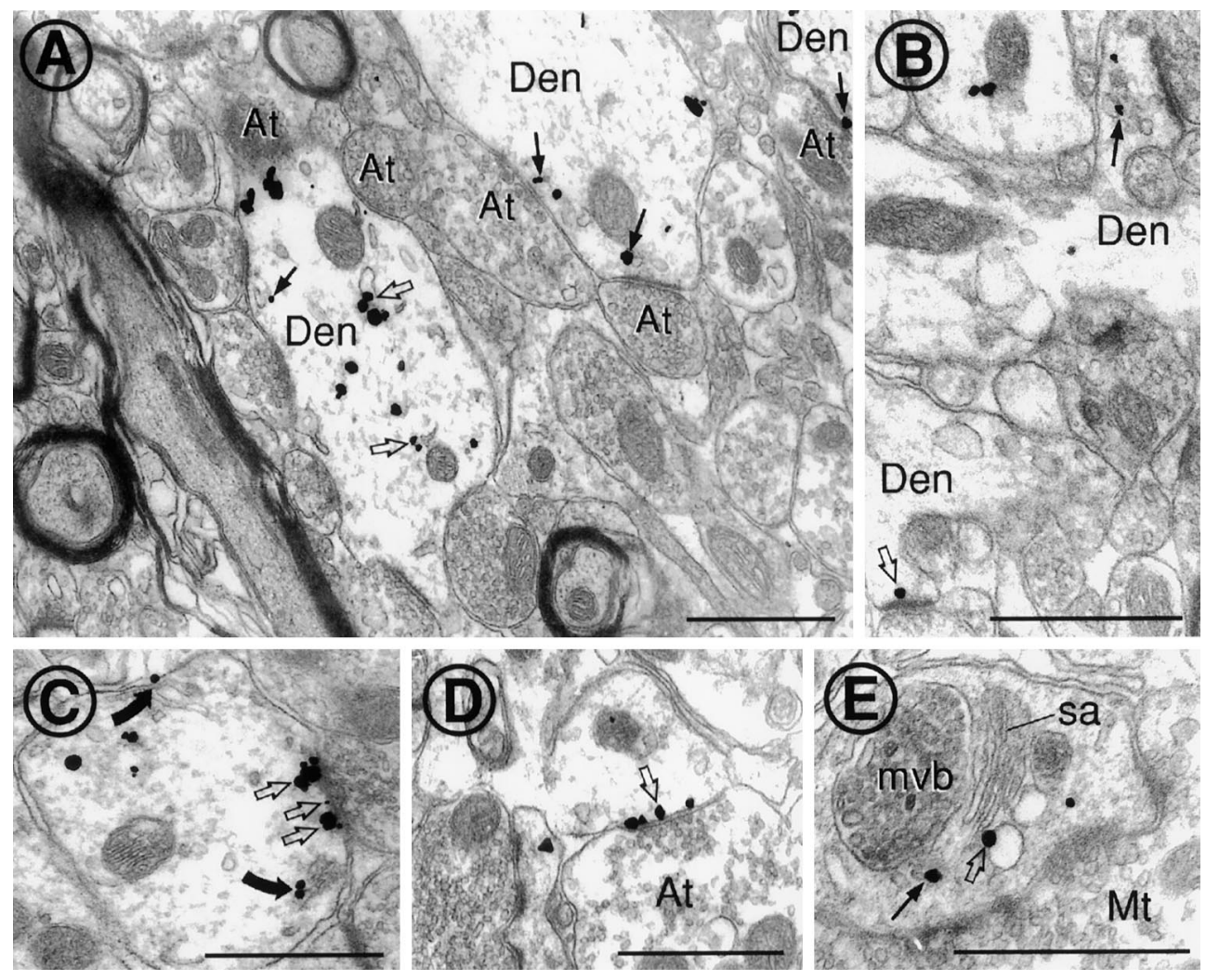

Figure 9. Ultrastructural study of RP3 in the dental hilus, using immunogold labeling. $A$, Two large dendrites (Den) were contacted by multiple axon terminals. In the dendrite cytoplasm RP3-derived immunogold particles were associated with the tubular-like smooth endoplasmic reticulum (open arrows). Many of these RP3-positive membranes (arrows) also were located near the synaptic contacts to axon terminals (At). A few axon terminals shown in this micrograph lacked RP3 labeling. B, RP3 immunoreactivity was associated with the smooth endoplasmic reticulum (arrow) and the PSD (open arrow) of two spines arising from separate dendrites (Den). $C$, In a dendritic profile the RP3-derived immunogold particles (open arrows) were clustered on the postsynaptic element apposing from an axon terminal. Smooth endoplasmic reticulum (curved arrows) also was labeled by RP3 immunogold particles $D$, In a dendritic profile several RP3-derived immunogold particles (open arrow) were concentrated at the postsynaptic elements that were in direct contact with a large axon terminal $(A t) . E$, In a spine head the gold particles indicative of RP3 were localized to a typical multi-cisternae spine apparatus (sa; open arrow) and smooth endoplasmic reticulum (arrow). $m v b$, Multivesicular body; Mt, mossy fiber terminal. Scale bars, $0.5 \mu \mathrm{m}$.

perform not only the general dynein housekeeping functions but also their own specific functions.

\section{DISCUSSION}

This study demonstrates that Tctex-1 and RP3 are expressed differentially at the cellular, subcellular, and developmental levels in the rat hippocampal formation. Immunoprecipitation experiments further suggest that Tctex-1 and RP3 are assembled in mutually exclusive dynein complexes in the brain. Finally, the preferential enrichment of Tctex-1 and RP3 in the presynaptic and the postsynaptic terminals, respectively, in the hippocampal neurons suggests that cytoplasmic dynein may have undiscovered functions at synapses.

\section{Subunit heterogeneity of the cytoplasmic dynein complex}

MT-based translocation is involved in a wide array of cellular events (Hirokawa et al., 1998). The two major classes of MT motors (i.e., kinesin and dynein) apparently use two different strategies to perform their diverse functions. A large superfamily of genes encodes the kinesins, whereas the number of genes encoding dynein subunits is comparatively low. Thus, other mechanisms probably exist to regulate the occurrence and specificity of various dynein activities.

Subunit heterogeneity could be one such mechanism. To date, isoforms of almost all dynein subunits have been reported (Gill et al., 1994; Pfister et al., 1996; Naisbitt et al., 2000). The expression of many of these subunit isoforms is regulated in a tissue-specific and/or development-specific manner, indicating that dynein is probably a heterogeneous population of molecules in vivo (Roux et al., 1994; Criswell et al., 1996; Nobuyuki et al., 1997; King et al., 1998). However, these tissue-based studies cannot reveal how the different subunit isoforms are assembled in vivo at the cellular and subcellular levels. Previously, immunofluorescent staining has suggested that the two DLCs, Tctex-1 and RP3, have distinct subcellular distributions in normal rat kidney (NRK) fibroblasts (King et al., 1998). The present report provides further biochemical and immunocytochemical evidence demonstrating that alternative $14 \mathrm{kDa}$ DLCs indeed are assembled in distinct populations of dynein complexes and that the differentially composed dynein complexes are expressed in a spatially and temporally regulated manner.

Increasing evidence suggests that several dynein subunits are able to bind to specific receptors on cargoes and act as adapters in linking dynein complexes to selected cargoes (see introductory remarks). Thus, dynein complexes with different compositions might perform different dynein-mediated functions, depending on their specific cargo recognition abilities. Our previous results have suggested that Tctex-1 and RP3 are highly selective in cargo binding: Tctex-1, but not RP3, binds to rhodopsin (Tai et al., 1999). We also have found that Tctex-1 and RP3 compete with 
one another in binding to the dynein complex. Moreover, ectopic overexpression of RP3 displaces Tctex-1 from the dynein complex, and this DLC alteration is accompanied by a change in the polarized transport of rhodopsin (Tai et al., 2001). These observations suggest that dynein complexes with different compositions can exhibit different properties, such as cargo specificity.

It has been shown that in NRK fibroblasts a subset of Tctex-1 does not associate with the intermediate chain, indicating the existence of a free DLC pool (Tai et al., 1998). It is currently unclear whether the free form or the complexed form of DLC or both mediate the cargo binding in vivo. It is probable that the Tctex-1 and RP3 immunoreactivities seen in this study represent a mixture of the free and complexed forms of DLC. Future work, such as colocalization studies with dynein heavy chain to determine the distribution of different forms of DLC in situ, would be useful in modeling the dynein cargo-docking mechanism.

\section{Expression of Tctex-1 in young neurons}

Both Tctex-1 and RP3 mRNA levels are regulated developmentally. Tctex-1 messages are abundant in fetal brain but are downregulated in the adult brain (Nobuyuki et al., 1997), whereas RP3 is regulated in the opposite manner (Roux et al., 1994). Our finding that, at the cellular level, RP3 immunoreactivity was prominent whereas Tctex-1 immunoreactivity was scant in mature granule cells agrees with these reports. The selectively abundant Tctex-1 expression in embryonic brain and young postmitotic neurons suggests that Tctex-1-containing dynein is involved in neurogenic events, such as synaptic reorganization, axonal guidance, or axonal outgrowth. The observations that flies with mutations in various subunits of the dynein-dynactin complex are defective in giant fiber synapse formation (Allen et al., 1999) and sensory neuron axonal pathfinding (Phillis et al., 1996; Reddy et al., 1997) support the role of dynein in these processes.

Synaptic development and maturation require target-derived long-distance retrograde signaling, which relays information from the distal axon terminal back to the cell body (Davis and Murphey, 1994). One classic example of retrograde signal propagation is the return of activated nerve growth factor-TrkA receptor complex from axon terminals back to the soma (Riccio et al., 1997). Because cytoplasmic dynein has a recognized role in general retrograde transport, it is the prime candidate motor protein for such retrograde signaling. Recent evidence has confirmed that this synapse-to-nucleus transport is MT-dependent (Watson et al., 1999). Moreover, Tctex-1 interacts with TrkA in vitro (Yano et al., 2001). Finally, both Tctex-1 and TrkA (BarkerGibb et al., 2001) show a similar predominant axon terminal localization in the hippocampal formation. Collectively, these results suggest that Tctex-1 may function as an adapter linking the Trk receptor to the dynein complex for retrograde transport and, hence, influence synaptogenesis.

\section{Tctex-1 localization at presynaptic neuronal sites}

Generally, it is believed that the MT-based motor proteins are used for long-range transport, whereas actin-based transport is used for local movement at the cell periphery (Goode et al., 2000). Consistently, the role of MT-dependent trafficking at the distal extremities of axons has been questionable because most EM studies have not shown MTs in these regions. However, a few ultrastructural studies have demonstrated that a subset of MTs indeed extends into the cortical cytoskeletal matrix of axon terminals, and sometimes these MTs are decorated by arrays of synaptic vesicles (Gray, 1978; Gray et al., 1982; Westrum et al.,
1983). This evidence is supported further by the presence of the KIF3A kinesin, a MT-based motor, on vesicles docked at the synaptic ribbon (specialized active zone) in photoreceptor synapses (Muresan et al., 1999).

The presence of MT and MT-based motors could have several, not necessarily mutually exclusive, functions in axon terminals. On one hand, Tctex-1-positive vesicles simply may represent recycling membranes destined for retrograde transport in the process of loading onto MT tracks. On the other hand, the frequent association of Tctex-1 immunoreactivity at the synaptic active zone and the known role of dynein in transport between early and late endosomes (Aniento et al., 1993) suggest that Tctex-1/dynein may be participating in the recycling of synaptic vesicles within axon terminals. Several additional lines of indirect evidence also support this possibility. Cytoplasmic dynein has been identified as a peripheral membrane protein of synaptic vesicles (Lacey and Haimo, 1992), although small synaptic vesicles themselves do not undergo retrograde transport (Kelly, 1988; Sudhof and Jahn, 1991). Moreover, Tctex-1 interacts directly with a synaptic vesicle-associated protein Doc2 (Nagano et al., 1998), which has been shown to play an important role in $\mathrm{Ca}^{2+}$ dependent neurotransmitter secretion (Orita et al., 1997; Verhage et al., 1997; Mochida et al., 1998). Finally, Tctex-1 is present on smooth endoplasmic reticulum, a putative membrane-recycling compartment (Peters et al., 1991), in mossy fiber terminals.

\section{Perikaryon and postsynaptic localization of RP3 and its possible functions}

As discussed above, a few Tctex-1-interacting proteins have been isolated, whereas no RP3-interacting protein has been reported yet. Thus, the cargo molecule and the specific cellular function of RP3 remain unknown. The RP3 association with Golgi complex, TGN, mitochondria, multivesicular body, and nematosome-like inclusion in somata suggest that RP3 is involved in docking some of these organelles to the dynein complex for the MT-based transport.

Furthermore, the localization of RP3 in several postsynaptic elements suggests that it could be involved in trafficking membranous constituents to and from the postsynaptic plasma membrane. First, RP3 immunoreactivity is present on the superficial membrane of the multivesicular body, which is an endosomalrecycling compartment (Schmied and Holtzman, 1987). The putative role of RP3 in membrane recycling at the postsynaptic site may represent a counterpart of Tctex-1 at the presynaptic site. Second, RP3 immunoreactivity is associated with the spine apparatus, which has been proposed to be a repository site for synaptic membrane components and even a postsynaptic translation site for spine modification (Jones and Harris, 1995). Third, RP3 immunoreactivity is affiliated with PSDs, a known postsynaptic structure to which many structural proteins, receptors, and signaling proteins are anchored (Kennedy, 19 97). PSDs are also highly plastic structures (Okabe et al., 1999); the dense matrix within PSDs changes significantly during development and in response to stimulation (Rees et al., 1985). Recent evidence suggests that multiple PSD-95 family proteins associate with MTs directly or indirectly (Brenman et al., 1998; Passafaro et al., 1999). Moreover, the $8 \mathrm{kDa}$ DLC is enriched at the PSD, and it binds to the PSD-95/GKAP complex (Naisbitt et al., 2000).

Membrane remodeling of dendritic spines is critical for longterm potentiation. This has been evidenced by activity-related changes in the dendritic structures, density, and protein compositions (Fifkova and Van Harreveld, 1977; Moser et al., 1994). 
RP3-mediated dynein activity could be functionally important for synaptic plasticity, for example, by actively recruiting and moving specific constituents to and/or from the postsynaptic plasma membranes.

\section{REFERENCES}

Allen MJ, Shan X, Caruccio P, Froggett SJ, Moffat KG, Murphey RK (1999) Targeted expression of truncated glued disrupts giant fiber synapse formation in Drosophila. J Neurosci 19:9374-9384.

Altman J, Das GD (1965) Autoradiographic and histological evidence of postnatal hippocampal neurogenesis in rats. J Comp Neurol 124:319-335

Amaral DG (1978) A Golgi study of cell types in the hilar region of the hippocampus in the rat. J Comp Neurol 182:851-914.

Aniento F, Emans N, Griffiths G, Gruenberg J (1993) Cytoplasmic dynein-dependent vesicular transport from early to late endosomes. J Cell Biol 123:1373-1387.

Baas PW, Deitch JS, Black MM, Banker GA (1988) Polarity orientation of microtubules in hippocampal neurons: uniformity in the axon and nonuniformity in the dendrite. Proc Natl Acad Sci USA 85:8335-8339.

Barker-Gibb AL, Dougherty KD, Einheber S, Drake CT, Milner TA (2001) Hippocampal tyrosine kinase A receptors are restricted primarily to presynaptic vesicle clusters. J Comp Neurol 430:182-199.

Bayer SA, Yackel JW (1982) Neurons in the rat dentate gyrus granular layer substantially increase during juvenile and adult life. Science 216:890-892.

Bowman AB, Patel-King RS, Benashski SE, McCaffery JM, Goldstein LSB, King SM (1999) Drosophila roadblock and Chlamydomonas LC7: a conserved family of dynein-associated proteins involved in axonal transport, flagellar motility, and mitosis. J Cell Biol 146:165-179.

Brenman JE, Topinka JR, Cooper EC, McGee AW, Rosen J, Milroy T, Ralston HJ, Bredt D (1998) Localization of postsynaptic density-93 to dendritic microtubules and interaction with microtubule-associated protein 1A. J Neurosci 18:8805-8813.

Cameron HA, Woolley CS, McEwen BS, Gould E (1993) Differentiation of newly born neurons and glia in the dentate gyrus of the adult rat. Neuroscience 56:337-344.

Chan J, Aoki C, Pickel VM (1990) Optimization of differential immunogold-silver and peroxidase labeling with maintenance of ultrastructure in brain sections before plastic embedding. J Neurosci Methods 33:113-127.

Chuang JZ, Milner TA, Zhu M, Sung CH (1999) A 29 kDa intracellular chloride channel p64H1 is associated with large dense-core vesicles in rat hippocampal neurons. J Neurosci 19:2919-2928.

Commons KG, Milner TA (1995) Ultrastructural heterogeneity of enkephalin-containing terminals in the rat hippocampal formation. J Comp Neurol 358:324-342.

Criswell PS, Ostrowski LE, Asai DJ (1996) A novel cytoplasmic dynein heavy chain: expression of DHC1b in mammalian epithelial cells. J Cell Sci 109:1891-1898.

Davis GW, Murphey RK (1994) Long-term regulation of short-term transmitter release properties: retrograde signaling and synaptic development. Trends Neurosci 17:9-13.

Echeverri C, Paschal B, Vaughan K, Vallee R (1996) Molecular characterization of the $50-\mathrm{kD}$ subunit of dynactin reveals function for the complex in chromosome alignment and spindle organization during mitosis. J Cell Biol 132:617-633.

Fifkova E, Van Harreveld A (1977) Long-lasting morphological changes in dendritic spines of dentate granular cells following stimulation of the entorhinal area. J Neurocytol 6:211-230.

Frotscher M, Seress L, Schwerdtfeger WK, Buhl E (1991) The mossy cells of the fascia dentata: a comparative study of their fine structure and synaptic connections in rodents and primates. J Comp Neurol 312:145-163.

Gibbons BH, Asai DJ, Tang WJ, Hays TS, Gibbons IR (1994) Phylogeny and expression of axonemal and cytoplasmic dynein genes in sea urchins. Mol Biol Cell 5:57-70.

Gill S, Cleveland D, Schroer T (1994) Characterization of DLC-A and DLC-B, two families of cytoplasmic dynein light chain subunits. Mol Biol Cell 5:645-654.

Goode BL, Drubin DG, Barnes G (2000) Functional cooperation between the microtubule and actin cytoskeletons. Curr Opin Cell Biol 12:63-71.

Gray EG (1978) Synaptic vesicles and microtubules in frog motor endplates. Proc R Soc Lond [Biol] 203:219-227.

Gray EG, Westrum LE, Burgoyne RD, Barron J (1982) Synaptic organization and neuron microtubule distribution. Cell Tissue Res 226:579-588

Gueneau G, Privat A, Drouet J, Court L (1982) Subgranular zone of the dentate gyrus of young rabbits as a secondary matrix. A high-resolution autoradiographic study. Dev Neurosci 5:345-358.

Hirokawa N, Noda Y, Okada Y (1998) Kinesin and dynein superfamily proteins in organelle transport and cell division. Curr Opin Cell Biol 10:60-73.

Jones DJ, Harris RJ (1995) An analysis of contemporary morphological concepts of synaptic remodeling in the CNS: perforated synapses revisited. Neurosci Rev 6:177-219.

Kaplan MS, Hinds JW (1977) Neurogenesis in adult rat: EM analysis of light radioautographs. Science 197:1092-1094.

Kelly RB (1988) The cell biology of the nerve terminal. Neuron 1:431-438.

Kennedy MB (1997) The postsynaptic density at glutamatergic synapses. Trends Neurosci 20:264-268.

King SM, Barbarese E, Dillman 3rd JF, Patel-King RS, Carson JH, Pfister KK (1996a) Brain cytoplasmic and flagellar outer arm dyneins share a highly conserved $M_{\mathrm{r}} 8000$ light chain. J Biol Chem 271:19358-19366.

King SM, Dillman 3rd JF, Benashski SE, Lye RJ, Patel-King RS, Pfister KK (1996b) The mouse $t$-complex-encoded protein Tctex-1 is a light chain of brain cytoplasmic dynein. J Biol Chem 271:32281-32287.

King SM, Barbarese E, Dillman 3rd JF, Benashski SE, Do KT, PatelKing RS, Pfister KK (1998) Cytoplasmic dynein contains a family of differentially expressed light chains. Biochemistry 37:15033-15041.

Lacey ML, Haimo LT (1992) Cytoplasmic dynein is a vesicle protein. J Biol Chem 267:4793-4798.

Mikkonen M, Soininen H, Pitkanen A (1997) Distribution of parvalbumin-, calretinin-, and calbindin-D28k-immunoreactive neurons and fibers in the human entorhinal cortex. J Comp Neurol 388:64-88.

Milner TA, Veznedaroglu E (1992) Ultrastructural localization of neuropeptide Y-like immunoreactivity in the rat hippocampal formation. Hippocampus 2:107-126.

Minturn J, Geschwind D, Fryer H, Hockfield S (1995a) Early postmitotic neurons transiently express TOAD-64, a neural specific protein. J Comp Neurol 355:369-379.

Minturn JE, Fryer HJ, Geschwind DH, Hockfield S (1995b) TOAD-64, a gene expressed early in neuronal differentiation in the rat, is related to unc-33, a C. elegans gene involved in axon outgrowth. J Neurosci 15:6757-6766.

Mochida S, Orita S, Sakaguchi G, Sasaki T, Takai Y (1998) Role of the Doc2 $\alpha$-Munc13-1 interaction in the neurotransmitter release process. Proc Natl Acad Sci USA 95:11418-11422.

Mok YK, Lo KW, Zhang M (2001) Structure of tctex-1 and its interaction with cytoplasmic dynein intermediate chain. J Biol Chem 276:14067-14074.

Moser MB, Trommald M, Andersen P (1994) An increase in dendritic spine density on hippocampal CA1 pyramidal cells following spatial learning in adult rats suggests the formation of new synapses. Proc Natl Acad Sci USA 91:12673-12675.

Muresan V, Lyass A, Schnapp BJ (1999) The kinesin motor KIF3A is a component of the presynaptic ribbon in vertebrate photoreceptors. J Neurosci 19:1027-1037.

Nagano F, Orita S, Sasaki T, Naito A, Sakaguchi G, Maeda M, Watanabe $\mathrm{T}$, Kominami E, Uchiyama Y, Takai Y (1998) Interaction of Doc2 with tctex-1, a light chain of cytoplasmic dynein. Implication in dyneindependent vesicle transport. J Biol Chem 273:30065-30068.

Naisbitt S, Valtschanoff J, Allison DW, Sala C, Kim E, Craig AM, Weinberg RJ, Sheng M (2000) Interaction of the postsynaptic density95/guanylate kinase domain-associated protein complex with a light chain of myosin-V and dynein. J Neurosci 20:4524-4534.

Nelson WJ (1992) Regulation of cell surface polarity from bacteria to mammals. Science 258:948-955.

Nobuyuki K, Mishina M, Yagi T (1997) Molecular cloning of fynassociated molecules in the mouse central nervous system. J Neurosci Res 48:407-424.

Okabe S, Kim H-D, Miwa A, Kuriu T, Okado H (1999) Continual remodeling of postsynaptic density and its regulation by synaptic activity. Nat Neurosci 2:804-811.

Orita S, Naito A, Sakaguchi G, Maeda M, Igarashi H, Sasaki T, Takai Y (1997) Physical and functional interactions of Doc2 and Munc13 in $\mathrm{Ca}^{2+}$-dependent exocytotic machinery. J Biol Chem 272:16081-16084.

Parent JM, Yu TW, Leibowitz RT, Geschwind DH, Sloviter RS, Lowenstein DH (1997) Dentate granule cell neurogenesis is increased by seizures and contributes to aberrant network reorganization in the adult rat hippocampus. J Neurosci 17:3727-3738.

Passafaro M, Sala C, Niethammer M, Sheng M (1999) Microtubule binding by CRIPT and its potential role in the synaptic clustering of PSD-95. Nat Neurosci 2:1063-1069.

Patton PE, McNaughton B (1995) Connection matrix of the hippocampal formation. I. The dentate gyrus. Hippocampus 5:245-286.

Peters A, Palay SL, Webster HD (1991) The fine structure of the nervous system, 3rd Ed. New York: Oxford UP.

Pfister K, Salata M, Dillman J, Torre E, Lye J (1996) Identification and developmental regulation of a neuron-specific subunit of cytoplasmic dynein. Mol Biol Cell 7:331-343.

Phillis R, Statton D, Caruccio P, Murphey RK (1996) Mutations in the 8 
$\mathrm{kDa}$ dynein light chain gene disrupt sensory axon projections in the Drosophila imaginal CNS. Development 122:2955-2963.

Reddy S, Jin P, Trimarchi J, Caruccio P, Phillis R, Murphey RK (1997) Mutant molecular motors disrupt neural circuits in Drosophila. J Neurobiol 33:711-723.

Rees S, Guldner FH, Aitkin L (1985) Activity-dependent plasticity of postsynaptic density structure in the ventral cochlear nucleus of the rat. Brain Res 325:370-374.

Ribak CE, Seress L, Amaral DG (1985) The development, ultrastructure, and synaptic connections of the mossy cells of the dentate gyrus. J Neurocytol 14:835-857.

Riccio A, Pierchala BA, Ciarallo CL, Ginty DD (1997) An NGF-TrkAmediated retrograde signal to transcription factor CREB in sympathetic neurons. Science 277:1097-1100.

Roux A-F, Rommens J, McDowell C, Anson-Cartwright L, Bell S, Schappert K, Fishman GA, Musarella M (1994) Identification of a gene from Xp21 with similarity to the tctex-1 gene of the murine $t$ complex. Hum Mol Genet 3:257-263.

Schmied R, Holtzman E (1987) A phosphatase activity and a synaptic vesicle antigen in multivesicular bodies of frog retinal photoreceptor terminals. J Neurocytol 16:627-637.

Scott BW, Wang S, Burnham WM, De Boni U, Wojtowicz JM (1998) Kindling-induced neurogenesis in the dentate gyrus of the rat. Neurosci Lett 248:73-76.

Seress L, Nitsch R, Leranth C (1993) Calretinin immunoreactivity in the monkey hippocampal formation. I. Light and EM characteristics and colocalization with other calcium-binding protein. Neuroscience 55:775-796.

Sudhof TC, Jahn R (1991) Proteins of synaptic vesicles involved in exocytosis and membrane recycling. Neuron 6:665-677.

Sung C-H, Davenport CM, Nathans J (1993) Rhodopsin mutations responsible for autosomal dominant retinitis pigmentosa: clustering of functional classes along the polypeptide chain. J Biol Chem 268:26645-26649.

Tai AW, Chuang J-Z, Sung C-H (1998) Localization of Tctex-1, a cytoplasmic dynein light chain, to the Golgi apparatus and evidence for dynein complex heterogeneity. J Biol Chem 273:19639-19649.

Tai AW, Chuang J-Z, Bode C, Wolfrum U, Sung C-H (1999) Rhodop- sin's carboxy-terminal cytoplasmic tail acts as a membrane receptor for cytoplasmic dynein by binding to the dynein light chain Tctex-1. Cell 97:877-887.

Tai AW, Chuang J-Z, Sung C-H (2001) Cytoplasmic dynein regulation by subunit heterogeneity and its role in apical transport. J Cell Biol, in press.

Tanaka Y, Zhang Z, Hirokawa N (1995) Identification and molecular evolution of new dynein-like protein sequences in rat brain. J Cell Sci 108:1883-1893.

Vaisberg EA, Grissom PM, McIntosh JR (1996) Mammalian cells express three distinct dynein heavy chains that are localized to different cytoplasmic organelles. J Cell Biol 133:831-842.

Vallee RB, Bloom GS (1991) Mechanisms of fast and slow axonal transport. Annu Rev Neurosci 14:59-92.

Vallee RB, Shpetner HS (1990) Motor proteins of cytoplasmic microtubules. Annu Rev Biochem 59:909-932

Vaughan KT, Vallee RB (1995) Cytoplasmic dynein binds dynactin through a direct interaction between the intermediate chains and p150 Glued. J Cell Biol 131:1507-1516.

Verhage M, de Vries KJ, Roshol H, Burbach JP, Gispen WH, Sudhof TC (1997) DOC2 proteins in rat brain: complementary distribution and proposed function as vesicular adapter proteins in early stages of secretion. Neuron 18:453-461.

Veznedaroglu E, Milner TA (1992) Elimination of artificial labeling of hippocampal mossy fibers seen following pre-embedding immunogoldsilver technique by pretreatment with zinc chelator. Microsc Res Tech 23:100-101.

Wang S, Scott BW, Wojtowicz JM (2000) Heterogenous properties of dentate granule neurons in the adult rat. J Neurobiol 42:248-257.

Watson FL, Heerssen HM, Moheban DB, Lin MZ, Sauvageot CM, Bhattacharyya A, Pomeroy SL, Segal RA (1999) Rapid nuclear responses to target-derived neurotrophins require retrograde transport of ligand-receptor complex. J Neurosci 19:7889-7900.

Westrum LE, Gray EG, Burgoyne RD, Barron J (1983) Synaptic development and microtubule organization. Cell Tissue Res 231:93-102.

Yano H, Lee F, Kong H, Chuang J-Z, Arevalo JC, Perez P, Sung C-H, Chao MV (2001) Association of Trk neurotrophin receptors with a component of the cytoplasmic dynein motor. J Neurosci 21:RC125. 